\title{
LncRNA MEG3 Reduces Hippocampal Neuron Apoptosis via the PI3K/AKT/mTOR Pathway in a Rat Model of Temporal Lobe Epilepsy
}

This article was published in the following Dove Press journal: Neuropsychiatric Disease and Treatment

\author{
Hongyan Zhang' \\ Jiuyun $\mathrm{TaO}^{2}$ \\ ShuXia Zhang ${ }^{3}$ \\ XinXin Lv ${ }^{4}$
}

'Department of Pediatrics, The First People's Hospital of Jinan, Jinan, Shandong 2500I I, People's Republic of China; ${ }^{2}$ Department of Surgery I, Chiping County People's Hospital, Liaocheng, Shandong 252100, People's Republic of China; ${ }^{3}$ Department of Obstetrics, Zhangqiu People's Hospital of Jinan City, Jinan, Shandong 250200, People's Republic of China; ${ }^{4}$ Department of Pediatrics, Jining First People's Hospital, Jining, Shandong 272000, People's Republic of China
Correspondence: XinXin Lv Department of Pediatrics, Jining First People's Hospital, No. 6 Jiankang Road, Jining, Shandong 272000, People's Republic of China

Email luxinxin|xx12@I63.com
Purpose: Temporal lobe epilepsy (TLE) is a common neurological disorder, which is characterized by recurrent spontaneous seizures. Exploring the mechanisms of epileptogenesis has been considered as a priority. The aim of this study is to investigate the effects of LncRNA MEG3 in spontaneous recurrent epileptiform discharges (SREDs) and rats with TLE.

Methods: Rat model of TLE was produced by intraperitoneal injection of lithium chloride and pilocarpine. Rat hippocampal neuronal model of SREDs was established by $\mathrm{Mg}^{2+}$-free treatment. MEG3 was overexpressed by transfection of AAV-MEG3 in TLE and SREDs model. The expression of MEG3, interleukin-1 $\beta$ (IL-1 $\beta$ ), interleukin-6 (IL-6) and recombinant human tumor necrosis factor-alpha (TNF- $\alpha$ ) was detected by reverse transcriptionquantitative polymerase chain reaction (RT-qPCR). Malondialdehyde (MDA) content and superoxide dismutase (SOD) activity were detected by corresponding kit. The apoptosis of hippocampal neurons was detected by terminal deoxynucleotidyl transferase transfer-mediated dUTP nick end-labeling (TUNEL) assay and flow cytometry. The expression of proteins related to apoptosis (Caspase-3, Bax, and Bcl-2) and the PI3K/AKT/mTOR pathway was detected by Western blot.

Results: MEG3 expression was downregulated in SREDs and rats with TLE. Overexpression of MEG3 reduced the expression of IL-1 $\beta$, IL-6, and TNF- $\alpha$, MDA content, apoptosis rate of hippocampal neuron, increased SOD activity, and inhibited the PI3K/AKT/ mTOR pathway in rats with TLE. In addition, overexpression of MEG3 enhanced cell viability and inhibited apoptosis through the activation of the PI3K/AKT/mTOR pathway in SREDs.

Conclusion: MEG3 reduced proinflammatory cytokines, oxidative stress, and apoptosis rate of hippocampal neuron and enhanced cell viability through the activation of the PI3K/AKT/ mTOR pathway in SREDs and rats with TLE. Our findings may contribute to find a new therapeutic target for the treatment of epilepsy.

Keywords: LncRNA MEG3, temporal lobe epilepsy, PI3K/AKT/mTOR pathway, apoptosis, inflammatory cytokines

\section{Introduction}

Epilepsy is a common neurological disease, affecting more than 50 million people worldwide. ${ }^{1}$ Its clinical feature is a sudden abnormal discharge of neurons that leads to short-term nerve dysfunction and behavior abnormalities including awareness, sensation, and movement disorders. ${ }^{2,3}$ Epidemiological data show that the incidence of epilepsy in the elderly is high, and its etiology is complex and diverse. 
Approximately $25 \%$ of patients with epilepsy are refractory temporal lobe epilepsy (TLE), and the effects of various antiepileptic drugs are not satisfactory. ${ }^{4}$ Moreover, long-term administration may make patients more vulnerable to a range of serious adverse reactions such as damage to the hematopoietic system, severe dysfunction of the liver or worsening cognitive dysfunction. ${ }^{5}$ Therefore, exploring the mechanisms and finding a novel therapeutic approach for epilepsy are of great priority.

Long non-coding RNA (lncRNA) is a kind of noncoding RNA (ncRNA) with a length greater than 200nt, and it does not have the ability to encode proteins. ${ }^{6}$ In recent years, studies have found that IncRNA can participate in the regulation of gene expression and plays a role in some molecular processes such as transcriptional activity, mRNA stability, and mRNA translation. ${ }^{7}$ Furthermore, some studies have indicated that IncRNAs are specifically expressed in the brain tissue and involved in nervous system diseases, including cerebral infarction, gliomas, and epilepsy. ${ }^{8-10}$ Maternally expressed gene 3, also known as MEG3, is a well-known tumor suppressor gene. Overexpression of MEG3 can inhibit proliferation and promote apoptosis in U251 and U87 cells (human glioma cell lines). ${ }^{11}$ However, the biological role of MEG3 in the occurrence and progression of epilepsy remains unknown.

At present, most scholars believe that the pathogenesis of epilepsy is related to the changes in ion channels, immunity, neurotransmitters, glial cells, and the abnormal discharge of neurons. ${ }^{12}$ Many studies have indicated that multiple signaling pathways may be related to epilepsy, including $\mathrm{PI} 3 \mathrm{~K} / \mathrm{AKT} / \mathrm{mTOR}$, Notch, and Wnt/ $\beta$-catenin signaling pathways. ${ }^{13-15}$ Viscomi et al have found that $\mathrm{CB} 2 \mathrm{R}$ protects neuron axons from damage by regulating the PI3K/AKT pathway. ${ }^{16}$ We speculated that MEG3 may participate in disease progression through this pathway. However, there is no evidence to prove this mechanism.

In this study, we investigated the effects of MEG3 on proinflammatory cytokines, apoptosis of hippocampal neurons, oxidative stress, and the PI3K/AKT/mTOR pathway in a rat model of TLE. More importantly, the regulation of this pathway was investigated in the spontaneous recurrent epileptiform discharges (SREDs) model. The results of our study may provide a new direction and target for the treatment of TLE.

\section{Materials and Methods}

\section{Experimental Animals}

Male Sprague Dawley (SD) rats (6-8 weeks, $180 \pm 220$ g, from Shandong Experimental Animal Research Center in Jinan, Shandong, China) were used in this study. All animals were housed in a room $\left(23^{\circ} \mathrm{C} \pm 2^{\circ} \mathrm{C}, 12 \mathrm{~h}\right.$ light/ dark cycle) and had free access to food and water. All experiments were approved by the Animal Ethics Committee of Animal Center of Jining First People's Hospital and complied with the National Institute of Health Guide for the Care and Use of Laboratory Animals.

\section{Establishment of TLE Model}

The rats were randomly divided into Control $(\mathrm{n}=10)$, Sham $(n=10)$ and TLE groups $(n=50)$. TLE model was produced by intraperitoneal injection of lithium chloride (LiCl, $125 \mathrm{mg} / \mathrm{kg}$; Sigma-Aldrich, Merck KgaA, Darmstadt, Germany) followed by intraperitoneal injection of pilocarpine $(20 \mathrm{mg} / \mathrm{kg}, 18-20 \mathrm{~h}$ later; Sigma-Aldrich). After the first injection of pilocarpine for $30 \mathrm{~min}$, the behavior of rats was observed and graded according to Racine criteria, wherein successful modeling is achieved for a score of $4-5 .^{17}$ If no epileptic seizures were observed, $10 \mathrm{mg} / \mathrm{kg}$ pilocarpine administration was repeated every 30 min until reaching Racine IV-V grade. Similarly, pilocarpine injection was administered for a maximum of six times. After continuous epileptic activity for $30 \mathrm{~min}$, rats received diazepam $(10 \mathrm{mg} / \mathrm{kg}$; intraperitoneal injection; Shharvest, Shanghai, China) to inhibit epilepsy activity. Rats in the Sham group were intraperitoneally injected with the same volume of $0.9 \%$ saline (125 mg/kg; Shharvest), and the other operations were the same as TLE group. After modeling for 28 days, all rats were euthanized using pentobarbital sodium $(30 \mathrm{mg} / \mathrm{kg}$, Sigma-Aldrich) anesthesia, and the hippocampus was collected, fixed in $4 \%$ paraformaldehyde or frozen in liquid nitrogen until further tests. Meanwhile, rats in the Control group did not receive any treatment.

\section{MEG3 Overexpression Experiments}

Rats in TLE group were randomly divided into TLE ( $\mathrm{n}=$ 10), TLE + NC $(\mathrm{n}=10)$ and TLE + MEG3 group $(\mathrm{n}=10)$. The AAV9 vectors carrying MEG3 (AAV9-MEG3) and empty adeno-associated virus (AAV) vector (AAV-NC) were constructed by Shanghai Genechem Co., LTD. (Shanghai, China). After modeling for $2 \mathrm{~h}$, the right dorsal hippocampus (3.12 $\mathrm{mm}$ posterior to the bregma, $3.0 \mathrm{~mm}$ 
lateral to the midline and $3.4 \mathrm{~mm}$ ventral to the bregma) and ventral hippocampus $(5.04 \mathrm{~mm}$ posterior to the bregma, $5.0 \mathrm{~mm}$ lateral to the midline, and $6.4 \mathrm{~mm}$ ventral to the bregma) of rats in TLE + NC and TLE + MEG3 group received $6 \mu \mathrm{L}$ corresponding AAV vector $(3 \mu \mathrm{L}$ at each location in the dorsal-ventral plane) through a microsyringe (Legato 130, KD Scientific, USA) at a speed of $0.2 \mu \mathrm{L} / \mathrm{min}$. After injection, the pipette stayed in place for $5 \mathrm{~min}$ to prevent backflow of viral particles.

\section{Reverse Transcription-Quantitative Polymerase Chain Reaction (RT-qPCR)} TRIzol reagent (Thermo Fisher Scientific, Inc., Waltham, MA, USA) was used to extract the total RNA from cells and hippocampus tissues of rats. The RevertAid First Strand cDNA synthesis kit (Thermo Fisher Scientific) was used to synthesize cDNA. qPCR was performed with SuperReal PreMix Plus (SYBR Green, TIANGEN) on ABI Mx3000P qPCR system (Stratagene; Agilent Technologies, Inc., Santa Clara, CA, USA) at $95^{\circ} \mathrm{C}$ for $30 \mathrm{~s}, 95^{\circ} \mathrm{C}$ for $15 \mathrm{~s}$ and $60^{\circ} \mathrm{C}$ for $30 \mathrm{~s}$ for a total of 40 cycles. The primer sequences (Sangon Biotech Co. Ltd, Shanghai, China) of RT-qPCR are shown in Table 1. Relative mRNA expression was normalized to GAPDH using the $2^{-\Delta \Delta \mathrm{Ct}}$ method.

\section{Detection of MDA Content and SOD Activity}

The hippocampus tissue was homogenized in ice-cold $0.9 \%$ normal saline. After centrifugation $\left(2500 \times \mathrm{g}, 4^{\circ} \mathrm{C}\right.$, $20 \mathrm{~min}$ ), the supernatant was used to measure the activity of MDA and SOD using the malondialdehyde (MDA) kit and superoxide dismutase (SOD) kit (Nanjing Jiancheng Bioengineering Research Institute, Nanjing, China) according to the manufacturer's protocols.

Table I The Sequences of Primers

\begin{tabular}{|l|l|}
\hline Primers & Sequences $\mathbf{( 5}^{\prime}$ - $\mathbf{3}^{\prime}$ ) \\
\hline MEG3-F & CTGCCCATCTACACCTCACG \\
MEG3-R & CTCTCCGCCGTCTGCGCTAGGGGCT \\
IL-I $\beta-F$ & ACAAGGAGAGACAAGCAACGACAA \\
IL-I $\beta-R$ & TTTCCATCTTCTTCTTTGGGTATTG \\
IL-6-F & AGACTTCACAGAGGA TACCACCCAC \\
IL-6-R & CAATCAGAATTGCCATTGCACAA \\
TNF- $\alpha-F$ & GCCACCACGCTCTTCTGTC \\
TNF- $\alpha-R$ & GCTACGGGCTTGTCACTCG \\
GAPDH-F & GTCAACGGATTTGGTCTGTATT \\
GAPDH-R & AGTCTTCTGGGTGGCAGTGAT \\
\hline
\end{tabular}

\section{Terminal Deoxynucleotidyl Transferase} Transfer-Mediated dUTP Nick End-labeling (TUNEL) Assay

Apoptosis of hippocampal neurons was detected by the in situ cell death detection kit (Roche, Palo Alto, CA). After dehydration, the hippocampus tissue was sliced into sections $(5 \mu \mathrm{m})$, incubated with a TUNEL reaction mixture for $1 \mathrm{~h}$ at $37^{\circ} \mathrm{C}$ in the dark. Next, the sections were stained with $0.1 \mu \mathrm{g} / \mathrm{mL}$ DAPI (Roche). Finally, the images of CA1 regions were taken under a fluorescence microscope (Olympus, Japan). The apoptotic rate was calculated as follows: number of TUNEL-positive cells/number of total cells $\times 100 \%$.

\section{Western Blot Analysis}

Radio-immunoprecipitation assay buffer (Shanghai Beyotime Biotechnology Co., Ltd, Shanghai, China) was used to extract total protein from cells and the hippocampus tissue. Butyleyanoacrylate (BCA) method was used to detect the concentration of proteins. These were separated via $10 \%$ sodium dodecyl sulfate-polyacrylamide gel electrophoresis (SDS-PAGE) for $2.5 \mathrm{~h}$ and transferred to polyvinylidene fluoride (PVDF) membranes (Millipore Corp, Massachusetts, Italy). The membranes were blocked with TBST containing 5\% skim milk for $1 \mathrm{~h}$ at room temperature. Next, the membranes were incubated with primary antibodies (Caspase-3, 14220; Bax, 14796; PI3K, 4249; AKT, 4691; p-AKT, 4060; mTOR, 2983; p-mTOR, 5536; GAPDH (control), 5174; 1:1000, Cell Signaling Technology, USA. Bcl-2, ab196495, 1:1000, Abcam, USA) overnight at $4^{\circ} \mathrm{C}$. The membranes were incubated with secondary antibody (1:5000, Santa Cruz, CA, USA) for $1 \mathrm{~h}$ at room temperature. The bands were illuminated by chemiluminescence reagents (ECL, Thermo Scientific) and analyzed via Quantity One software (BioRad, USA).

\section{Establishment of Hippocampal Neuronal SREDs Model and Grouping}

The hippocampal neurons of SD rats were cultured within $24 \mathrm{~h}$ after birth. On the 7th day of culture, the hippocampal neurons were divided into Control, SREDs, SREDs+NC, SREDs+MEG3 and SREDs+MEG3+LY294002 groups. The Control group was replaced with the normal cell culture medium for $3 \mathrm{~h}$ and then exchanged for maintenance medium. The SREDs group was replaced with the $\mathrm{Mg}^{2+}$-free medium for $3 \mathrm{~h}$ and then exchanged for maintenance medium. The hippocampal neurons in SREDs+NC 
group were incubated with $\mathrm{Mg}^{2+}$-free medium for $3 \mathrm{~h}$, exchanged for maintenance medium, and then transfected with AAV-NC. The hippocampal neurons in SREDs +MEG3 group were incubated with $\mathrm{Mg}^{2+}$-free medium for $3 \mathrm{~h}$, exchanged for maintenance medium, and then transfected with AAV-MEG3. The hippocampal neurons in SREDs+MEG3+LY294002 group were incubated with maintenance medium containing $1 \mu \mathrm{moL} / \mathrm{L}$ LY294002 (PI3K inhibitor, Sigma) for $24 \mathrm{~h}$, incubated with $\mathrm{Mg}^{2+}$ free medium for $3 \mathrm{~h}$, and then transfected with AAV-MEG3. After transfection for $48 \mathrm{~h}$, the hippocampal neurons were collected for further experiments.

\section{Cell Viability Assay}

Cell counting kit-8 (CCK-8, Dojindo Laboratories, Japan) was used to measure cell viability. After transfection for $48 \mathrm{~h}$, the cells were incubated with $10 \mu \mathrm{L} \mathrm{CCK} 8$ solution for $2 \mathrm{~h}$ at $37^{\circ} \mathrm{C}$. Subsequently, Multiskan MK-3 (Thermo Fisher) was used to measure the absorbance at $450 \mathrm{~nm}$. Cell viability was expressed as percentages of the control values.

\section{Flow Cytometry Assay}

After transfection for $48 \mathrm{~h}$, the cells were harvested and resuspended in $1 \times$ Annexin $\mathrm{V}$ binding buffer $(500 \mu \mathrm{L})$. Next, the cells were incubated with Annexin V-FITC $(5 \mu \mathrm{L})$ and propidium iodide $(5 \mu \mathrm{L})$ for $15 \mathrm{~min}$ at room temperature without light. Finally, the apoptosis ratio was analyzed using FACScan flow cytometry (BD Biosciences).

\section{Statistical Analysis}

All experimental data were expressed as mean \pm standard deviation (SD) using Graphpad prism software for statistical analysis. Statistical significance was analyzed by oneway analysis of variance with Tukey's post hoc test among multiple groups, and the samples were normally distributed. Results showed that the difference was statistically significant $(\mathrm{P}<0.05)$.

\section{Results}

Downregulation of LncRNA MEG3, Upregulation of Proinflammatory Cytokine and Increase Oxidative Stress in Rats with TLE

In this study, TLE model was produced by intraperitoneal injection of lithium chloride and pilocarpine. As shown in Figure 1A, the Racine score of TLE group was higher than that of Control and Sham group $(\mathrm{P}<0.01)$, and the TLE group reached Racine IV-V grade on the 20th day. To explore the effects of MEG3 on TLE, RT-qPCR was used to detect MEG3 expression in the hippocampus of the Control, Sham, and TLE groups. Results showed that MEG3 expression in the hippocampus of TLE group was downregulated $(\mathrm{P}<0.01$, Figure $1 \mathrm{~B})$. We also detected the mRNA expression of proinflammatory cytokines (IL-1 $\beta$, IL-6, and TNF- $\alpha$ ) by RT-qPCR. As shown in Figure 1C-E, the mRNA expression of IL-1 $\beta$, IL- 6 , and TNF- $\alpha$ in the hippocampus of TLE group was upregulated $(\mathrm{P}<0.01)$. In addition, MDA content in this group was higher than that in the Control, Sham groups $(\mathrm{P}<0.01$, Figure $1 \mathrm{~F})$, while SOD activity was decreased $(\mathrm{P}<0.01$, Figure $1 \mathrm{G})$.

\section{The Apoptosis of the Hippocampal Neurons is Increased and the PI3K/AKT/ mTOR Pathway is Inhibited in Rats with} TLE

The apoptosis of hippocampal neurons was detected using the TUNEL assay. Results suggested that epilepsy modeling resulted in a greater apoptosis of hippocampal neurons in the CA1 region than that in the Control and Sham groups $(\mathrm{P}<0.01$, Figure 2A). Moreover, we examined the expression of proteins related to apoptosis (Caspase-3, Bax, and Bcl-2) using Western blot. In Figure 2B, epilepsy modeling increased the protein expression of Caspase- 3 and Bax, as well as decreased the protein expression of Bcl-2 in the hippocampus tissue of rats $(\mathrm{P}<0.01)$. We analyzed the expression of proteins related to the $\mathrm{PI} 3 \mathrm{~K} / \mathrm{AKT} / \mathrm{mTOR}$ pathway using Western blot to investigate whether the PI3K/AKT/mTOR pathway participates in the occurrence and progression of epilepsy. In Figure 2C, epilepsy modeling reduced the protein expression of PI3K, p-AKT/ $\mathrm{AKT}$, and $\mathrm{p}-\mathrm{mTOR} / \mathrm{mTOR}$ in the hippocampus tissue of rats $(\mathrm{P}<0.01)$.

\section{LncRNA MEG3 Ameliorates Progression of Epilepsy and Activates the PI3K/AKT/ mTOR Pathway}

In this study, we found that epilepsy modeling reduced MEG3 expression. Therefore, we applied an AAV delivery system to the overexpression of MEG3 and investigated the role of MEG3 in rats with TLE. In Figure 3A, the transfection of AAVMEG3 increased MEG3 expression in the hippocampus $(\mathrm{P}<0.01)$, indicating the success of the transfection. Next, the mRNA expression of proinflammatory cytokines (IL-1 $\beta$, IL-6, and TNF- $\alpha$ ) in the hippocampus was detected by RT-qPCR. 
A

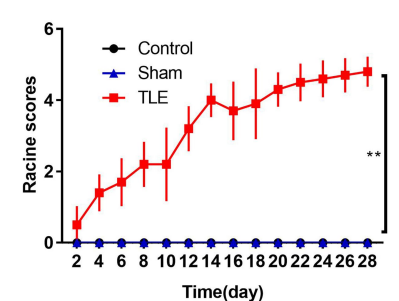

E

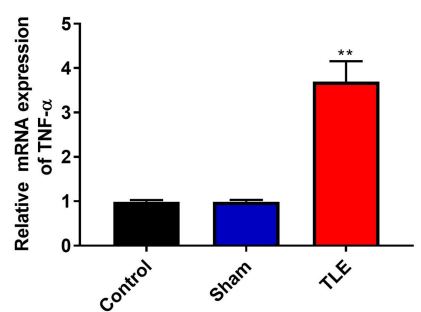

B

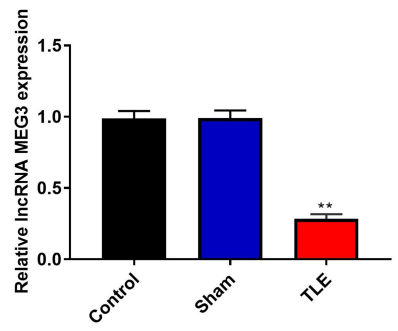

F

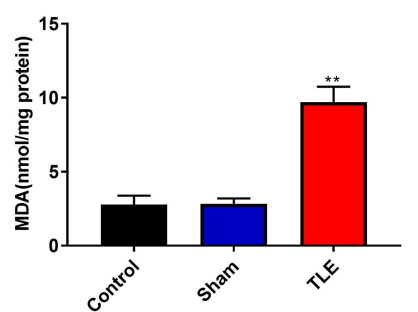

C

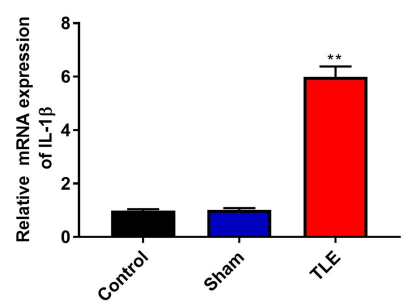

G

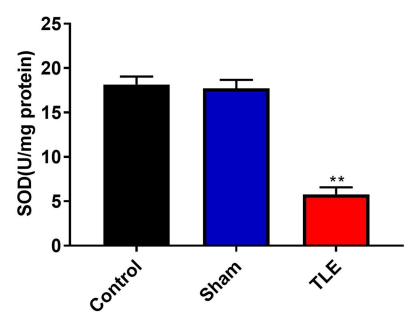

D

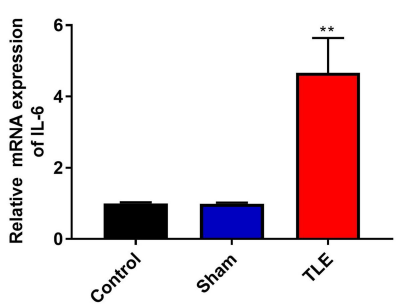

Figure I Downregulation of LncRNA MEG3, upregulation of proinflammatory cytokine and increase oxidative stress in rats with TLE. (A) The Racine score of Control, Sham and TLE groups. (B and E) RT-qPCR was used to detect the mRNA expression of MEG3 (B), IL-I $\beta$ (C), IL-6 (D) and TNF- $\alpha$ (E) in the hippocampus of Control, Sham and TLE groups. (F) Malondialdehyde (MDA) content in the hippocampus of Control, Sham and TLE groups. (G) Superoxide dismutase (SOD) activity in the hippocampus of Control, Sham and TLE groups. $* * \mathrm{P}<0.01$ vs Control and Sham groups.

A

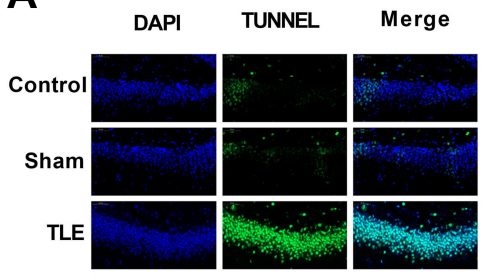

C

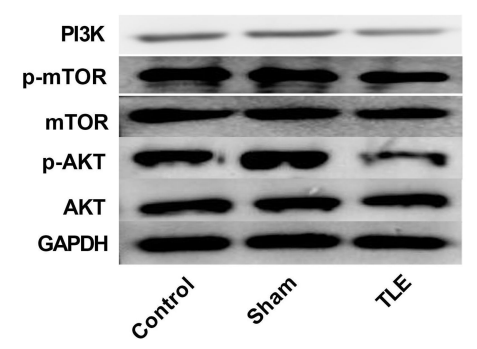

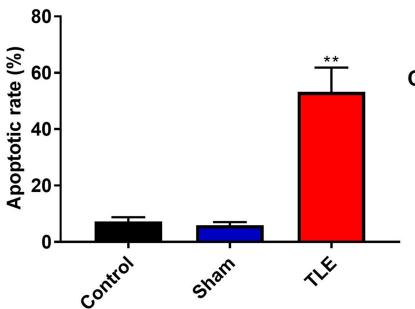

B

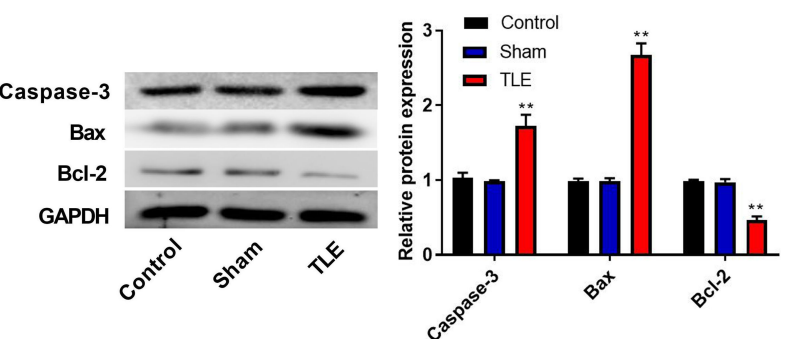

Figure 2 The apoptosis of the hippocampal neurons was increased and the PI3K/AKT/mTOR pathway was inhibited in rats with TLE. (A) The apoptosis of hippocampal neurons was detected using the TUNEL assay. (B) The expression of proteins related to apoptosis (Caspase-3, Bax and Bcl-2) was detected using Western blot. (C) The expression of proteins related to the PI3K/AKT/mTOR pathway was detected using Western blot. **P $<0.01$ vs Control and Sham groups.

Results showed that overexpression of MEG3 reduced the mRNA expression of IL-1 $\beta$, IL-6, and TNF- $\alpha$ in the hippocampus when compared with that in the TLE and TLE+NC groups $(\mathrm{P}<0.01$, Figure $3 \mathrm{~B}-\mathrm{D})$. We also examined the levels of oxidative stress markers (MDA and SOD). This revealed that overexpression of MEG3 decreased MDA content and increased SOD activity $(\mathrm{P}<0.01$, Figure $3 \mathrm{E}$ and $\mathrm{F})$. Furthermore, overexpression of MEG3 decreased the apoptosis of hippocampal neurons in CA1 region $(\mathrm{P}<0.01$, Figure $3 \mathrm{G})$. Meanwhile, overexpression of MEG3 decreased the protein expression of Caspase- 3 and Bax and increased the protein expression of $\mathrm{Bcl}-2$ in the hippocampus tissue of rats $(\mathrm{P}<0.01$, 

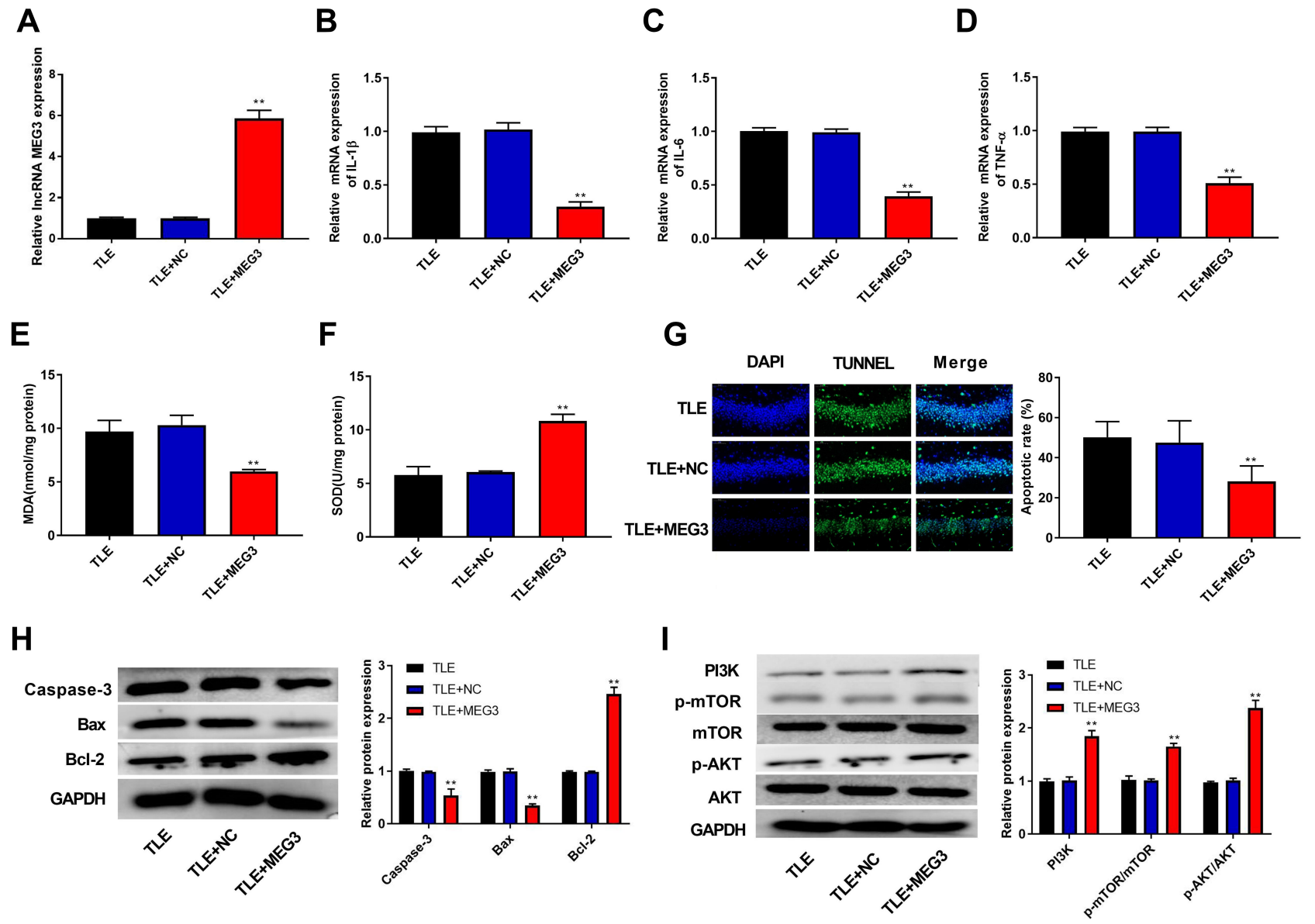

Figure 3 LncRNA MEG3 ameliorated progression of epilepsy and activated the PI3K/AKT/mTOR pathway. (A) RT-qPCR was used to detect the mRNA expression of MEG3 in the hippocampus tissue of rats. (B-D) RT-qPCR was used to detect the mRNA expression of IL-I $\beta$ (B), IL-6 (C) and TNF- $\alpha$ (D) in the hippocampus tissue of rats. (E) Malondialdehyde (MDA) content in the hippocampus tissue of rats. (F) Superoxide dismutase (SOD) activity in the hippocampus tissue of rats. (G) The apoptosis of hippocampal neurons was detected using TUNEL assay. $(\mathbf{H})$ The expression of proteins related to apoptosis (Caspase-3, Bax and Bcl-2) was detected using Western blot. (I) The expression of proteins related to the PI3K/AKT/mTOR pathway was detected using Western blot. **P $<0.01$ vs TLE and TLE+NC group.

Figure $3 \mathrm{H})$. In addition, the protein expression of $\mathrm{PI} 3 \mathrm{~K}$, $\mathrm{p}-\mathrm{AKT} / \mathrm{AKT}$, and $\mathrm{p}-\mathrm{mTOR} / \mathrm{mTOR}$ in the hippocampus tissue of rats was elevated $(\mathrm{P}<0.01$, Figure $3 \mathrm{I})$.

\section{LncRNA MEG3 Enhances Cell Viability, Inhibits Apoptosis and Activates the PI3K/ AKT/mTOR Pathway in SREDs}

To further assess the biological role of MEG3 in hippocampal neurons, we established a rat hippocampal neuronal model of SREDs by $\mathrm{Mg}^{2+}$-free treatment and transfected with AAV-MEG3 to increase MEG3 expression. As shown in Figure 4A, MEG3 expressions in SREDs, SREDs+NC, and SREDs+MEG3 groups were lower than that of the Control group $(\mathrm{P}<0.01)$, while the transfection of AAVMEG3 increased MEG3 expression $(\mathrm{P}<0.01)$. CCK-8 assay was used to measure hippocampal neurons viability. Results revealed that cell viabilities in SREDs, SREDs $+\mathrm{NC}$, and SREDs+MEG3 groups were lower than those in the Control group $(\mathrm{P}<0.01)$, but overexpression of MEG3 enhanced cell viability when compared with that in the SREDs and SREDs $+\mathrm{NC}$ groups $(\mathrm{P}<0.01$, Figure $4 \mathrm{~B})$. We also detected apoptosis of hippocampal neurons by flow cytometry. This suggested that apoptosis rates in SREDs, SREDs $+\mathrm{NC}$, and SREDs + MEG3 groups were higher than that of the Control group $(\mathrm{P}<0.05)$, but overexpression of MEG3 reduced apoptosis rate $(\mathrm{P}<0.05$, Figure $4 \mathrm{C})$. Meanwhile, protein expressions of Caspase- 3 and Bax in SREDs, SREDs+NC, and SREDs+MEG3 groups were greater than that in the Control group $(\mathrm{P}<0.05)$. Similarly, $\mathrm{Bcl}-2$ protein expression was decreased, but overexpression of MEG3 reduced the protein expression of Caspase- 3 and Bax and increased Bcl-2 protein expression $(\mathrm{P}<0.01$, Figure 4D). In addition, the expression of proteins related to the 

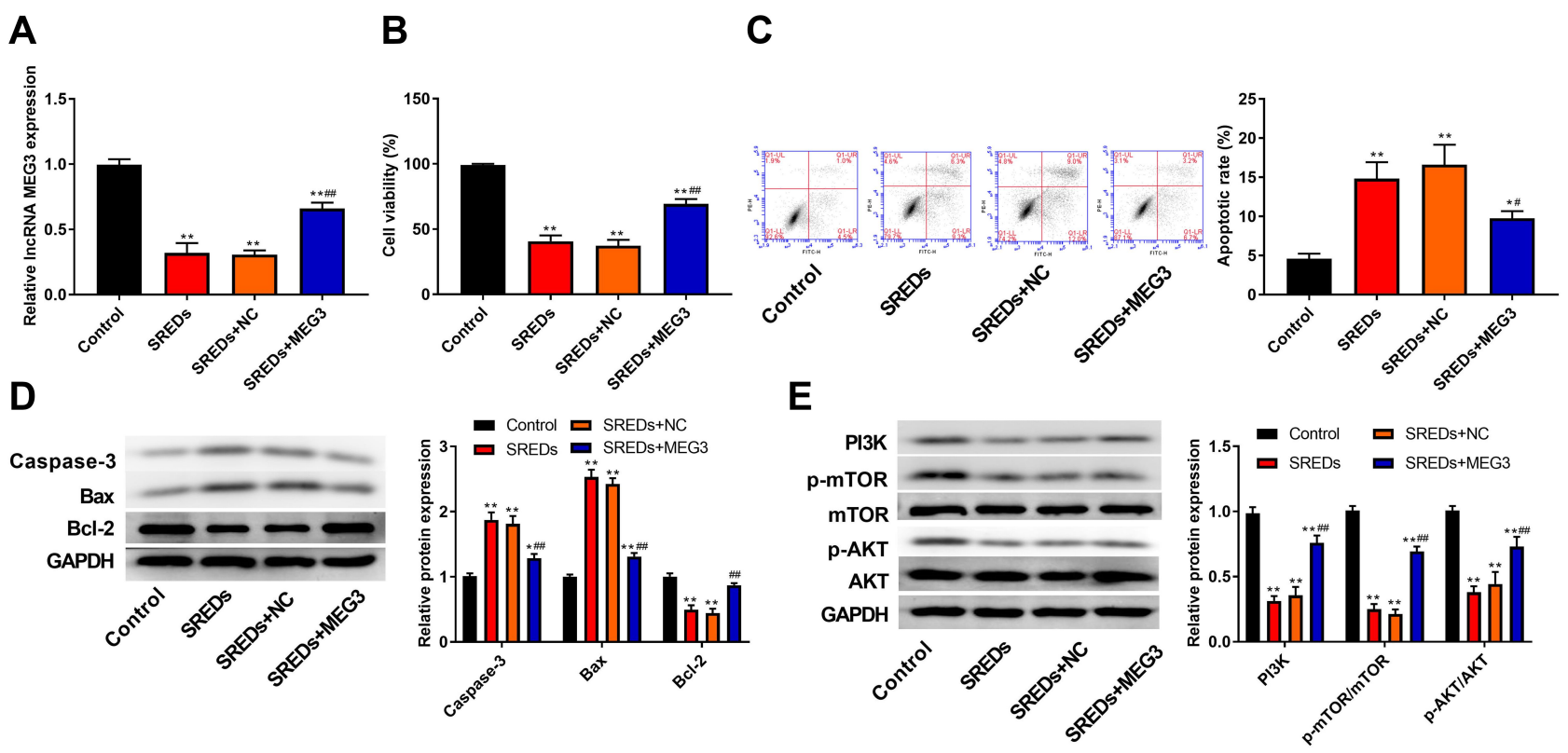

Figure 4 LncRNA MEG3 enhanced cell viability, inhibited apoptosis and activated the PI3K/AKT/mTOR pathway in SREDs. (A) RT-qPCR was used to detect the mRNA expression of MEG3 in the hippocampal neurons. (B) CCK-8 assay was used to measure hippocampal neurons viability. (C) Apoptosis of hippocampal neurons was detected using flow cytometry. (D) The expression of proteins related to apoptosis (Caspase-3, Bax and Bcl-2) in the hippocampal neurons was detected using Western blot. (E) The expression of proteins related to the PI3K/AKT/mTOR pathway in the hippocampal neurons was detected using Western blot. *P $<0.05$ and $* * \mathrm{P}<0.0 \mathrm{I}$ vs Control group, ${ }^{\#} \mathrm{P}<0.05$ and ${ }^{\# \#} \mathrm{P}<0.01$ vs SREDs and SREDs+NC groups.

$\mathrm{PI} 3 \mathrm{~K} / \mathrm{AKT} / \mathrm{mTOR}$ pathway was detected using Western blot. In Figure 4E, protein expressions of PI3K, p-AKT/AKT, and p-mTOR/mTOR in SREDs, SREDs+NC, and SREDs + MEG3 groups were less than that in the Control group $(\mathrm{P}<0.01)$, while overexpression of MEG3 increased the protein expression of PI3K, $\mathrm{p}-\mathrm{AKT} / \mathrm{AKT}$, and $\mathrm{p}-\mathrm{mTOR} / \mathrm{mTOR}$ $(\mathrm{P}<0.01)$.

\section{LncRNA MEG3 Enhances Cell Viability and Inhibits Apoptosis and Through the Activation the PI3K/AKT/mTOR Pathway in SREDs}

To further confirm whether MEG3 and the PI3K/AKT/ mTOR pathway interacted in the hippocampus and caused the occurrence and progression of epilepsy, PI3K inhibitor (LY294002) was used to treat the AAV-MEG3 transfected SREDs. In Figure $5 \mathrm{~A}$, protein expressions of $\mathrm{PI} 3 \mathrm{~K}$, $\mathrm{p}-\mathrm{AKT} / \mathrm{AKT}$, and $\mathrm{p}-\mathrm{mTOR} / \mathrm{mTOR}$ in SREDs+MEG3+ LY294002 group were lower than that in the SREDs + MEG3 group $(\mathrm{P}<0.01)$. This indicated that LY294002 reversed the promotion of $\mathrm{PI} 3 \mathrm{~K}, \mathrm{p}-\mathrm{AKT} / \mathrm{AKT}$, and $\mathrm{p}-\mathrm{mTOR} / \mathrm{mTOR}$ expression caused by overexpression of MEG3. Nonetheless, we detected the influences of LY294002 on hippocampal neurons viability and apoptosis. In Figure 5B and C, the addition of LY294002 reversed the MEG3-induced promotion on cell viability and inhibition on cell apoptosis $(\mathrm{P}<0.05)$, which suggested that the LncRNA MEG3 enhanced cell viability and inhibited apoptosis through activation of the PI3K/AKT/mTOR pathway in SREDs.

\section{Discussion}

The occurrence of epilepsy is closely related to hippocampus dysfunction. ${ }^{18}$ Hippocampal neurons cells are activated to form pathological neural signals, generate electrical signals and spread to the edge, and then induce epilepsy symptoms. ${ }^{19}$ There are many studies on the function of MEG3 in tumorigenesis. Moreover, recent studies have found that MEG3 is also implicated in nervous system diseases such as cerebral infarction, gliomas, and Huntington's disease. ${ }^{20-22}$ However, the biological role of MEG3 in epilepsy remains unclear. In this study, we established TLE model in rats by intraperitoneal injection of lithium chloride and pilocarpine. It was found that MEG3 expression was downregulated after modeling. Additionally, TLE modeling increased oxidative stress, expression of proinflammatory cytokine, and hippocampal neuronal apoptosis, which are typical during epilepsy.

LncRNA does not only regulate the gene expression of the physiological process and signal pathway in the nervous system but also regulate the expression of pro-inflammatory 
A

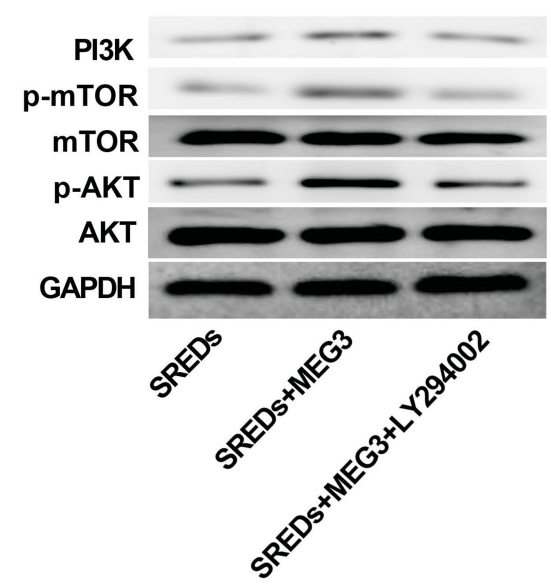

C
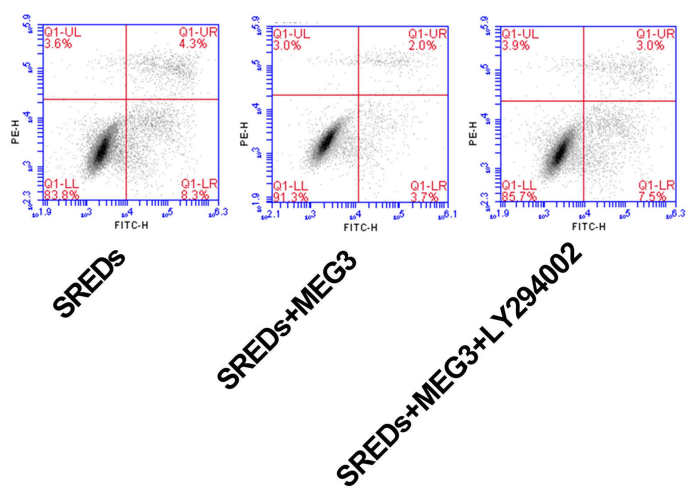

B

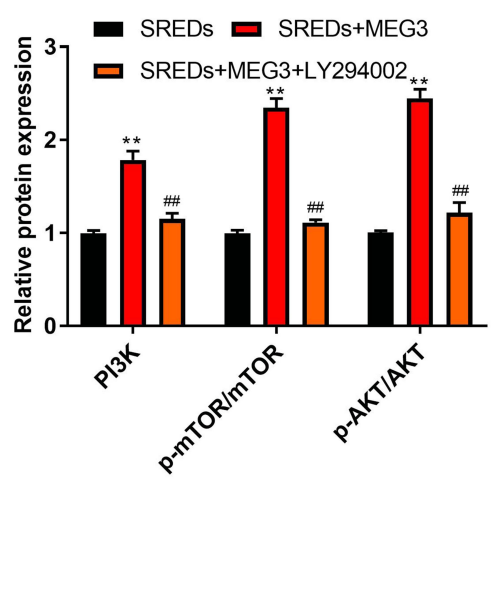

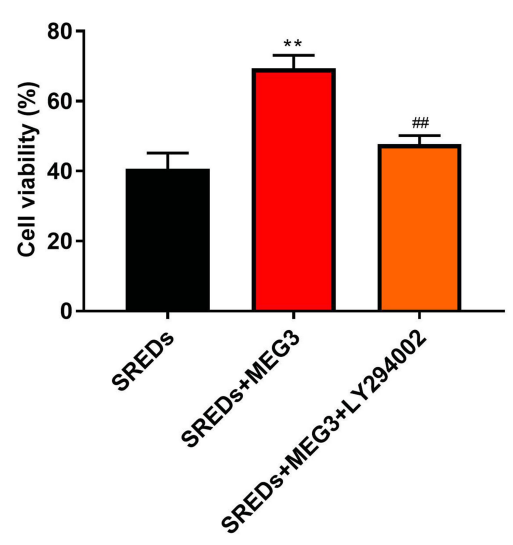

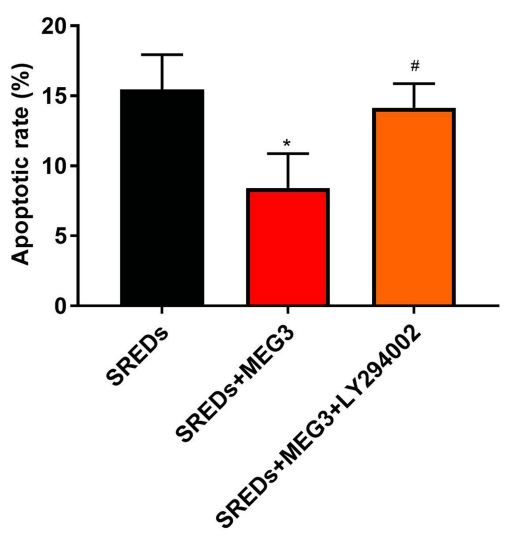

Figure 5 LncRNA MEG3 enhanced cell viability and inhibited apoptosis and through the activation of the PI3K/AKT/mTOR pathway in SREDs. (A) The expression of proteins related to the PI3K/AKT/mTOR pathway in the hippocampal neurons was detected using Western blot. (B) CCK-8 assay was used to measure hippocampal neurons viability. (C) Apoptosis of hippocampal neurons was detected using flow cytometry. ${ }^{* P}<0.05$ and ${ }^{* * P}<0.01$ vs SREDs group, ${ }^{\#} \mathrm{P}<0.05$ and ${ }^{\#} \mathrm{P}<0.0 \mathrm{I}$ vs SREDs +MEG3 group.

and anti-inflammatory cytokines. ${ }^{23}$ Vezzani et al have found that the expression of proinflammatory cytokines in glia of seizures is significantly increased in various experimental models. ${ }^{24}$ This suggested that the inflammatory response plays a very important role in the occurrence and development of epilepsy. In this study, we found that epilepsy modeling increased the expression of IL-1 $\beta$, IL-6, and TNF- $\alpha$ in the hippocampus. Moreover, overexpression of MEG3 reduced the expression of IL-1 $\beta$, IL-6, and TNF- $\alpha$ in the hippocampus of rats with TLE. These findings indicated that MEG3 had the function of inhibiting inflammation in rats with TLE.

Further studies have shown that a large number of free radicals and lipid peroxidation during metabolism are involved in the pathological process of epilepsy. ${ }^{25,26} \mathrm{MDA}$, which is an important free radical in the body, is considered to be a representative intermediate product of lipid peroxidation. This can also lead to dysfunction of cell metabolism and epilepsy. ${ }^{27} \mathrm{SOD}$, which is an important scavenger of free radical in the body, is also the key enzyme to scavenge MDA, which indirectly reflects the ability to scavenge oxygen-free radicals. ${ }^{28}$ Therefore, the detection of SOD activity and MDA content in the body can determine the degree of epilepsy nerve cell damage. In our study, it was found that epilepsy modeling increased MDA content and decreased SOD activity. In addition, overexpression of MEG3 reduced MDA content and increased SOD activity in the hippocampus of rats with TLE. These results suggested that MEG3 decreased oxidative stress in rats with TLE.

It is well known that both the onset and the persistent state of epilepsy can lead to neuronal damage. As such, apoptosis is an important cause of this damage. ${ }^{29,30}$ The occurrence of apoptosis is regulated by many genes (miRNA, Bcl-2, and Bax). ${ }^{13,31}$ The abnormal expression of apoptosis-associated 
genes is widely reported in TLE tissue and models. For example, Han et al have found that the protein expression of Caspase-3 is increased and Bcl-2 expression is decreased in KA-induced epileptic rats. ${ }^{32}$ In our study, we found that the apoptosis rate of hippocampal neuron was higher in rats with TLE and SREDs; meanwhile, the protein expression of Caspase-3 and Bax was upregulated and the protein expression of Bcl-2 was downregulated. Furthermore, we also demonstrated that overexpression of MEG3 decreased the apoptosis rate of the hippocampal neuron and protein expression of Caspase- 3 and Bax and increased the protein expression of Bcl-2 in rats with TLE and SREDs. The results showed that MEG3 inhibited apoptosis rate of hippocampal neuron.

In previous studies, the $\mathrm{PI} 3 \mathrm{~K} / \mathrm{AKT} / \mathrm{mTOR}$ pathway has been found to play an important role in nervous system diseases such as Parkinson's disease, neurodegenerative disease, and neuroblastoma. ${ }^{33-35}$ The PI3K/AKT/mTOR pathway is widely present in nerve cells and regulates the biological functions of nerve cell proliferation, apoptosis, differentiation, and metabolism. ${ }^{36,37}$ Duan et al have found that miRNA-155 promotes the occurrence of epilepsy through the PI3K/AKT/mTOR pathway. ${ }^{13} \mathrm{Lu}$ et al have reported that GLYX-13 relieves depressive-like behaviors in mice undergoing the forced swim test and upregulates the expression of phosphorylation of AKT and mTOR, while LY294002 abolished the antidepressantlike effects of GLYX-13. ${ }^{38}$ In this study, we found that overexpression of MEG3 increased the protein expression of PI3K, p-AKT/AKT, and p-mTOR/mTOR in rats with TLE and SREDs. Furthermore, the addition of PI3K inhibitor (LY294002) reversed the promotion of the PI3K/ $\mathrm{AKT} / \mathrm{mTOR}$ pathway and cell viability, as well as inhibition of cell apoptosis caused by overexpression of MEG3 in SREDs. These findings suggested that MEG3 enhanced cell viability and inhibited apoptosis through the activation of the PI3K/AKT/mTOR pathway in TLE.

However, there are still some limitations in our study. We did not detect the role of MEG3 in the other areas of the hippocampus associated with epilepsy (CA2, CA3, dentate gyrus), which might weaken our conclusion. In addition, the role of MEG3 on TLE rats at different time points should be studied. Therefore, further studies are required.

\section{Conclusion}

MEG3 expression was downregulated in SREDs and rats with TLE. Overexpression of MEG3 reduced proinflammatory cytokines, oxidative stress, and apoptosis rate of hippocampal neurons and inhibited the $\mathrm{PI} 3 \mathrm{~K} / \mathrm{AKT} / \mathrm{mTOR}$ pathway in rats with TLE. In addition, MEG3 enhanced cell viability and inhibited apoptosis through the activation of the PI3K/AKT/ mTOR pathway in SREDs. Our findings may contribute in developing a new therapeutic target for the treatment of epilepsy.

\section{Data Sharing Statement}

All data of this manuscript used to support the findings of this study may be released upon application to the correspondence author.

\section{Statement of Ethics}

This study was authorized by the ethical committee of Jining First People's Hospital and complied with the National Institute of Health Guide for the Care and Use of Laboratory Animals.

\section{Funding}

There is no funding to report.

\section{Disclosure}

The authors indicate no potential conflicts of interest.

\section{References}

1. Li X, Giri V, Cui Y, Yin M, Xian Z, Li J. LncRNA FTX inhibits hippocampal neuron apoptosis by regulating miR-21-5p/SOX7 axis in a rat model of temporal lobe epilepsy. Biochem Biophys Res Commun. 2019;512(1):79-86. doi:10.1016/j.bbrc.2019.03.019

2. Nevalainen O, Simola M, Ansakorpi H, et al. Epilepsy, excess deaths and years of life lost from external causes. Eur J Epidemiol. 2016;31 (5):445-453. doi:10.1007/s10654-015-0095-5

3. Lhatoo S, Noebels J, Whittemore V. Sudden unexpected death in epilepsy: identifying risk and preventing mortality. Epilepsia. 2015;56(11):1700-1706. doi:10.1111/epi.13134

4. Chen J, Liu XM, Yue X, Chen SZ. The clinical efficacy and safety of levetiracetam add-on therapy for child refractory epilepsy. Eur Rev Med Pharmacol Sci. 2016;20(12):2689-2694.

5. Mehla J, Reeta KH, Gupta P, Gupta YK. Protective effect of curcumin against seizures and cognitive impairment in a pentylenetetrazole-kindled epileptic rat model. Life Sci. 2010;87(19-22):596-603. doi:10.1016/j.lfs.2010.09.006

6. Wang HK, Yan H, Wang K, Wang J. Dynamic regulation effect of long non-coding RNA-UCA1 on NF-kB in hippocampus of epilepsy rats. Eur Rev Med Pharmacol Sci. 2017;21(13):3113-3119.

7. Zhou Y, Wang DL, Pang Q. Long noncoding RNA SPRY4-IT1 is a prognostic factor for poor overall survival and has an oncogenic role in glioma. Eur Rev Med Pharmacol Sci. 2016;20(14):3035-3039.

8. Qi X, Shao M, Sun H, Shen Y, Meng D, Huo W. Long non-coding RNA SNHG14 promotes microglia activation by regulating miR-1455p/PLA2G4A in cerebral infarction. Neuroscience. 2017;348:98-106. doi:10.1016/j.neuroscience.2017.02.002

9. Xu D, Liu R, Meng L, Zhang Y, Lu G, Ma P. Long non-coding RNA ENST01108 promotes carcinogenesis of glioma by acting as a molecular sponge to modulate miR-489. Biomed Pharmacother. 2018;100:20-28. doi:10.1016/j.biopha.2018.01.126 
10. Geng JF, Liu X, Zhao HB, Fan WF, Geng JJ, Liu XZ. LncRNA UCA1 inhibits epilepsy and seizure-induced brain injury by regulating miR-495/Nrf2-ARE signal pathway. Int J Biochem Cell Biol. 2018;99:133-139. doi:10.1016/j.biocel.2018.03.021

11. Wang P, Ren Z, Sun P. Overexpression of the long non-coding RNA MEG3 impairs in vitro glioma cell proliferation. $J$ Cell Biochem. 2012;113(6):1868-1874. doi:10.1002/jcb.24055

12. Kuznetsova LV, Vetrilé LA, Karpova MN. [Neuroimmunological aspects of the pathogenesis of epilepsy]. Usp Fiziol Nauk. 2014;45(3):3-22.

13. Duan W, Chen Y, Wang XR. MicroRNA-155 contributes to the occurrence of epilepsy through the PI3K/Akt/mTOR signaling pathway. Int J Mol Med. 2018;42(3):1577-1584.

14. Wu L, Li Y, Yu M, Yang F, Tu M, Xu H. Notch signaling regulates microglial activation and inflammatory reactions in a rat model of temporal lobe epilepsy. Neurochem Res. 2018;43(6):1269-1282. doi:10.1007/s11064-018-2544-5

15. Qu Z, Su F, Qi X, et al. Wnt/ $\beta$-catenin signalling pathway mediated aberrant hippocampal neurogenesis in kainic acid-induced epilepsy. Cell Biochem Funct. 2017;35(7):472-476. doi:10.1002/cbf.3306

16. Viscomi MT, Oddi S, Latini L, et al. Selective CB2 receptor agonism protects central neurons from remote axotomy-induced apoptosis through the PI3K/Akt pathway. $J$ Neurosci. 2009;29 (14):4564-4570. doi:10.1523/JNEUROSCI.0786-09.2009

17. Tamargo RJ, Rossell LA, Kossoff EH, Tyler BM, Ewend MG, Aryanpur JJ. The intracerebral administration of phenytoin using controlled-release polymers reduces experimental seizures in rats. Epilepsy Res. 2002;48(3):145-155. doi:10.1016/S0920-1211(01)00330-8

18. Chen SD, Chang AY, Chuang YC. The potential role of mitochondrial dysfunction in seizure-associated cell death in the hippocampus and epileptogenesis. J Bioenerg Biomembr. 2010;42(6):461-465. doi:10.1007/s10863-010-9321-8

19. Šapina L, Vuletić V, Lojen G, Janjetović Z, Vuković-Arar Z. Head trauma and posttraumatic epilepsy in Slavonski Brod, East Croatia, 1988-2008. Coll Antropol. 2014;38(3):1077-1079.

20. Zhan R, Xu K, Pan J, Xu Q, Xu S, Shen J. Long noncoding RNA MEG3 mediated angiogenesis after cerebral infarction through regulating p53/NOX4 axis. Biochem Biophys Res Commun. 2017;490 (3):700-706. doi:10.1016/j.bbrc.2017.06.104

21. Li J, Bian EB, He XJ, et al. Epigenetic repression of long non-coding RNA MEG3 mediated by DNMT1 represses the p53 pathway in gliomas. Int J Oncol. 2016;48(2):723-733. doi:10.3892/ijo.2015.3285

22. Chanda K, Das S, Chakraborty J, et al. Altered levels of long ncRNAs Meg3 and Neat1 in cell and animal models of Huntington's disease. RNA Biol. 2018;15(10):1348-1363. doi:10.1080/15476286.2018.1534524

23. Xu H, He L, Liu C, Tang L, Xu Y. LncRNA NONRATT021972 siRNA attenuates $\mathrm{P} 2 \mathrm{X} 7$ receptor expression and inflammatory cytokine production induced by combined high glucose and free fatty acids in PC12 cells. Purinergic Signal. 2016;12(2):259-268. doi:10.1007/s11302-016-9500-0

24. Vezzani A, Ravizza T, Balosso S, Aronica E. Glia as a source of cytokines: implications for neuronal excitability and survival. Epilepsia. 2008;49(2.x):24-32. doi:10.1111/j.1528-1167.2008.01 490.x

Neuropsychiatric Disease and Treatment

\section{Publish your work in this journal}

Neuropsychiatric Disease and Treatment is an international, peerreviewed journal of clinical therapeutics and pharmacology focusing on concise rapid reporting of clinical or pre-clinical studies on a range of neuropsychiatric and neurological disorders. This journal is indexed on PubMed Central, the 'PsycINFO' database and CAS, and
25. Frantseva MV, Perez Velazquez JL, Tsoraklidis G, et al. Oxidative stress is involved in seizure-induced neurodegeneration in the kindling model of epilepsy. Neuroscience. 2000;97(3):431-435. doi:10.1016/S0306-4522(00)00041-5

26. Pandey MK, Mittra P, Maheshwari PK. The lipid peroxidation product as a marker of oxidative stress in epilepsy. J Clin Diagn Res. 2012;6(4):590-592.

27. Ilhan A, Aladag MA, Kocer A, Boluk A, Gurel A, Armutcu F. Erdosteine ameliorates PTZ-induced oxidative stress in mice seizure model. Brain Res Bull. 2005;65(6):495-499. doi:10.1016/j. brainresbull.2005.02.027

28. Tejada S, Sureda A, Roca C, Gamundí A, Esteban S. Antioxidant response and oxidative damage in brain cortex after high dose of pilocarpine. Brain Res Bull. 2007;71(4):372-375. doi:10.1016/j. brainresbull.2006.10.005

29. Bengzon J, Mohapel P, Ekdahl CT, Lindvall O. Neuronal apoptosis after brief and prolonged seizures. Prog Brain Res. 2002;135:111-119.

30. Liou AK, Clark RS, Henshall DC, Yin XM, Chen J. To die or not to die for neurons in ischemia, traumatic brain injury and epilepsy: a review on the stress-activated signaling pathways and apoptotic pathways. Prog Neurobiol. 2003;69(2):103-142. doi:10.1016/ S0301-0082(03)00005-4

31. Huang Y, Liu X, Liao Y, Luo C, Zou D. MiR-181a influences the cognitive function of epileptic rats induced by pentylenetetrazol. Int J Clin Exp Pathol. 2015;8(10):12861-12868.

32. Han CL, Ge M, Liu YP, et al. Long non-coding RNA H19 contributes to apoptosis of hippocampal neurons by inhibiting let-7b in a rat model of temporal lobe epilepsy. Cell Death Dis. 2018;9 (6):018-0496. doi:10.1038/s41419-018-0496-y

33. Kawinthra K, Suriyat M, Khwanthana G, Permphan D. Comparative mRNA expression of eEF1A isoforms and a PI3K/Akt/mTOR pathway in a cellular model of Parkinson's disease. Parkinsons Dis. 2016;2016.

34. Heras-Sandoval D, Pérez-Rojas JM, Hernández-Damián J, PedrazaChaverri J. The role of PI3K/AKT/mTOR pathway in the modulation of autophagy and the clearance of protein aggregates in neurodegeneration. Cell Signal. 2014;26(12):2694-2701. doi:10.1016/j.cellsig.2014.08.019

35. Fulda S. The PI3K/Akt/mTOR pathway as therapeutic target in neuroblastoma. Curr Cancer Drug Targets. 2009;9(6):729-737. doi:10.2174/156800909789271521

36. Liu L, Sun T, Xin F, Cui W, Guo J, Hu J. Nerve growth factor protects against alcohol-induced neurotoxicity in PC12 cells via PI3K/Akt/mTOR pathway. Alcohol Alcohol. 2017;52(1):12-18. doi:10.1093/alcalc/agw077

37. Huang ZR, Chen HY, Hu ZZ, Xie P, Liu QH. PTEN knockdown with the Y444F mutant AAV2 vector promotes axonal regeneration in the adult optic nerve. Neural Regen Res. 2018;13(1):135-144. doi:10.4103/1673-5374.224381

38. Lu Y, Wang C, Xue Z, et al. PI3K/AKT/mTOR signaling-mediated neuropeptide VGF in the hippocampus of mice is involved in the rapid onset antidepressant-like effects of GLYX-13. Int J Neuropsychopharmacol. 2015;18(5). doi:10.1093/ijnp/pyu110 tely online and includes a very quick and fair peer-review system, which is all easy to use. Visit http://www.dovepress.com/testimonials.php to read real quotes from published authors.

\section{Dovepress}

